\title{
Microwave Assisted Synthesis of 3-Chloro-N-(2-(5-chloro-1-tosyl-1H- benzo [d] Imidazol-2-yl) ethyl)-N-Substituted Quinoxalin-2-Amine Derivatives Using DCQX
}

\author{
K.Shashikala ${ }^{1^{*}}$, E.Laxminarayana ${ }^{2}$, M.Ramesh ${ }^{3}$, M.Thirumala Chary ${ }^{4}$ \\ ${ }^{1}$ Geethanjali College of Engineering and Technology, Cheeryal(V), Keesara(M), Medchal Dt 501301, Telangana, India \\ ${ }^{2}$ Sreenidhi Institute of Science and Technology (Autonomous), Ghatkesaer, Hyderabad 501301, Telangana, India \\ ${ }^{3}$ Department of Biotechnology, Jawaharlal Nehru Technological University Kakinada, East Godavari Dt, Andhrapradesh 533003, India \\ ${ }^{4}$ Jawaharlal Nehru Technological University Hyderabad, Kukatpally, Hyderabad 500085, Telangana, India \\ E-mail: kala.shashi2010@gmail.com
}

\begin{abstract}
The microwave assisted synthesis of 3-Chloro-N-(2-(5-chloro-1-tosyl-1H-benzo [d] imidazol-2-yl) ethyl)$\mathrm{N}$-substituted quinoxalin-2-amine derivatives is described. 2,3-dichloro quinoxaline (DCQX), as a starting compound and propargyl bromide, as an efficient alkylating agent are used in the synthesis of $\mathrm{N}$-substituted quinoxalin-2-amine derivatives. We realized that microwave assisted synthesis is efficiently replacing conventional method of synthesis.

Keywords: 2,3-dichloroquinoxaline, quinoxaline, imidazoles, alkylating agent, microwave assisted synthesis
\end{abstract}

\section{Introduction}

There are several reported methods for the synthesis of quinoxaline-2-amine derivatives. Nevertheless, synthesis using 2,3-dichloroquinoxaline (DCQX) with nucleophilic species such as aryl amine has become a feasible substitute because of the presence of two chlorine atoms at $\mathrm{C} 2$ and $\mathrm{C} 3$ of DCQX. 2,3-dichloroquinoxaline (DCQX) is a reagent, extensively used as a synthetic intermediate in pharmaceutical industry as well as materials science ${ }^{[1,2]}$. Furthermore, this reagent is easily prepared from low-cost starting materials and commercially available.

One of the major advantages associated with the reactions of DCQX with nucleophiles is the possibility to control single or double substituted products. This exceptional feature of DCQX makes it significant in the synthesis of specific products that can be used in a variety of applications ${ }^{[3-7]}$. Propargyl bromide, an efficient alkylating agent is used for the $\mathrm{N}$-alkylation of aryl amides. It is also used in enyne metathesis of propargylic amines, propargylation of spiro ketones, synthesis of allylic alcohols and enone complexes ${ }^{[8,9]}$.

The effective approach for the synthesis of quinoxalin-2-amines is the reaction between 1,2-diamines with aldehydes and isocyanides using $\mathrm{CeO}_{2}$ nanoparticle catalyst. Also 3,4-dihydroquinoxalin-2-amines were synthesized by reactions between 1,2-diamines, ketones and isocyanides ${ }^{[10]}$.

Reaction between 2,3-dichloro quinoxaline and anilines is a convenient method for the preparation of N-aryl substituted 3-chloroquinoxalin-2-amines, particularly, 2-( $N$-aryl amino)-3-chloroquinoxalines that are further converted into $\mathrm{N}$-substituted 3-chloro-N-(2-(1-tosyl-1H-benzo [d]-imidazol-2-yl) ethyl) quinoxalin-2-amine ${ }^{[11]}$. This method is facilitated by $\mathrm{AlCl}_{3}$ on forming $\mathrm{C}-\mathrm{N}$ bond ${ }^{[1]}$. These target molecules were found to be potential inhibitors of phospho diesterase 4 (PDE-4) and have apoptosis inducing properties in an animal model (zebrafish) ${ }^{[12,13]}$. Further, the reaction is facilitated in more effective way using an alkylating agent, propargyl bromide.

\section{Results and discussions}

All the compounds were synthesized using microwave irradiation. The synthesis of new compounds is described according to synthetic Figure 1. Compound 2 was synthesized from the starting materials, 2,3-dichloroquinoxaline (DCQX) and aniline, substituted at $4^{\text {th }}$ position. Then compound 2 is irradiated with an alkylating agent, propargyl bromide in presence potassium carbonate and DMF to acquire compound 3 The final compound 3-Chloro-N-(2 (5-chloro-1-tosyl-1Hbenzo [d] imidazol-2-yl) ethyl)-N-substituted quinoxalin-2-amine (4) is obtained, when compound 3 was reacted with a

Copyright (C2020 K. Shashikala, et al.

DOI: https://doi.org/10.37256/ocp.122020287

This is an open-access article distributed under a CC BY license

(Creative Commons Attribution 4.0 International License)

https://creativecommons.org/licenses/by/4.0/ 
mixture containing $\mathrm{TSN}_{3}, \mathrm{CuI}, \mathrm{Et}_{3} \mathrm{~N}$ and $\mathrm{CH}_{3 \mathrm{C}} \mathrm{N}$.

The structures of all the synthesized compounds were confirmed by ${ }^{1} \mathrm{H}$ NMR, ${ }^{13} \mathrm{CNMR}$ and Mass spectral data. A triplet at 4.51-4.56ppm represents $\mathrm{CH}_{2} \mathrm{~N}$ group. A peak in the range of 3.61-4.73 ppm indicates the presence of methylene group. Aromatic protons were observed in expected regions in the range of 7-8ppm. The $[\mathrm{M}+\mathrm{H}]$ peaks of mass spectra of compounds are in agreement with their molecular formula.

\section{Experimental section}

All the chemicals used were purchased from Sigma-aldrich, Avra Laboratories. Solvents and reagents were obtained from commercial sources. Microwave assisted synthesis was carried out in BP090 Laboratory grade microwave oven. Melting Points are uncorrected and were determined using open capillary tubes in a bath of Sulphuric acid. TLC analyses were done on Merck pre-coated Plates (silica gel 60 F254) and spotting was done using Iodine / UV lamp. ${ }^{1} \mathrm{H}-\mathrm{NMR}$ and ${ }^{13} \mathrm{C}$ NMR were recorded in $\mathrm{CDCl}_{3}$ / DMSO-d6 using Varian MR-400MHZ and $100 \mathrm{MHz}$ spectrometer and TMS as a reference standard. Mass spectra were recorded on an Agilent-LCMS instrument.

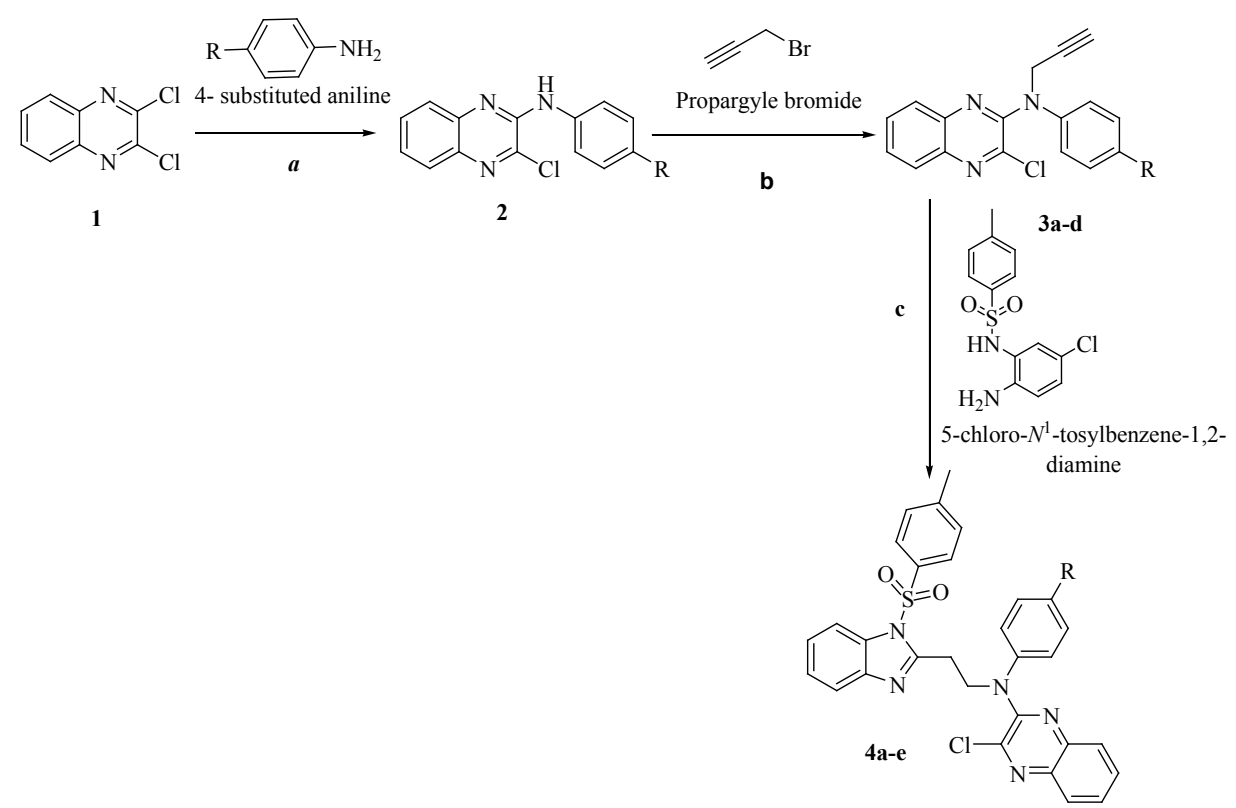

Figure 1. Synthesis of 3-Chloro-N-(2(5-chloro-1-tosyl-1H-benzo [d] imidazol-2-yl) ethyl)-N-p-tolyl quinoxalin-2-amine (4a-e)

Reagents and Conditions: (a) n-BuOH; M.W., $130^{\circ} \mathrm{C} ; 30$ min. (b) $\mathrm{K}_{2} \mathrm{CO}_{3}$; DMF; M.W.; $110^{\circ} \mathrm{C} ; 15 \mathrm{~min}$. (c) $\mathrm{TSN}_{3}$; $\mathrm{CuI} ; \mathrm{Et}_{3} \mathrm{~N} ; \mathrm{CH}_{3} \mathrm{CN} ; \mathrm{M} . \mathrm{W}$; $120^{\circ} \mathrm{C} ; 20 \mathrm{~min}$;

$3 \mathrm{a} \mathrm{R}=\mathrm{OCH}_{3} ; 3 \mathrm{~b} \mathrm{R}=\mathrm{Br} ; 3 \mathrm{c} \mathrm{R}=\mathrm{Cl} ; 3 \mathrm{~d} \mathrm{R}=\mathrm{F}$

$4 \mathrm{a} \mathrm{R}=\mathrm{CH}_{3} ; 4 \mathrm{~b} \mathrm{R}=\mathrm{OCH}_{3} ; 4 \mathrm{c} \mathrm{R}=\mathrm{Br} ; 4 \mathrm{~d} \mathrm{R}=\mathrm{Cl} ; 4 \mathrm{e}=\mathrm{F}$

Step 1: Synthesis of 3-Chloro-N-(p-tolyl)-N-(prop-2-ynyl) quinoxalin-2-amine (2)

2,3-dichloroquinoxaline (1) (1 mmol) and substituted aniline $(1 \mathrm{mmol})$ in $\mathrm{n}-\mathrm{BuOH}(2 \mathrm{~mL})$ was subjected to irradiation in microwave at $130^{\circ} \mathrm{C}$ for $30 \mathrm{~min}$. Reaction mixture was allowed to cooled to room temperature solvent was evaporated in reduced pressure. Crude material was purified by crystallization using ethanol to get compound 2 .

${ }^{1} \mathrm{HNMR}\left(400 \mathrm{MHz} \mathrm{CDCl}_{3}\right): \delta=7.95(\mathrm{~d}, 1 \mathrm{H}, \mathrm{J}=7.8 \mathrm{~Hz}, \mathrm{ArH}), 7.88(\mathrm{~d}, 1 \mathrm{H}, \mathrm{J}=7.8 \mathrm{~Hz}, \mathrm{ArH}), 7.67(\mathrm{t}, 1 \mathrm{H}, \mathrm{ArH}), 7.56(\mathrm{t}$, $1 \mathrm{H}, \mathrm{ArH}), 7.17(\mathrm{~d}, 2 \mathrm{H}, \mathrm{J}=7.6 \mathrm{~Hz}, \mathrm{ArH}), 7.03(\mathrm{~d}, 2 \mathrm{H}, \mathrm{J}=7.6 \mathrm{~Hz}, \mathrm{ArH}), 4.73\left(\mathrm{~d}, 2 \mathrm{H}, \mathrm{J}=2 \mathrm{~Hz},-\mathrm{CH}_{2}\right), 2.37\left(\mathrm{~s}, 3 \mathrm{H},-\mathrm{CH}_{3}\right), 2.19$ (t, 1H, C H);

${ }^{13} \mathrm{C}$ NMR $\left(100 \mathrm{MHz}, \mathrm{CDCl}_{3}\right): \delta=149,142,141,139,138,136,130,129,127,127,127,125,79,72,43,21 ;$ Mass: $\mathrm{m}$ $/ \mathrm{z}=308.6\left[\mathrm{M}^{+1}\right]$.

Step 2: General experimental procedure for the synthesis of (3a-d)

$\mathrm{K}_{2} \mathrm{CO}_{3}(0.7 \mathrm{mmol})$ was added to a solution of compound $2(0.47 \mathrm{mmol})$ and propargyl bromide ( $80 \%$ in toluene) $(0.94$ $\mathrm{mmol})$ in DMF $(1 \mathrm{~mL})$ was subjected to microwave irradiation at $110^{\circ} \mathrm{C}$ for $15 \mathrm{~min}$. After completion of reaction, reaction 
mixture was diluted with water $(15 \mathrm{~mL})$, extracted with EtOAc $(3 \times 8 \mathrm{~mL})$. The combined organic layer was washed with water $(2 \times 8 \mathrm{~mL})$, aqueous sodium chloride solution $(10 \mathrm{~mL})$, dried over anh. $\mathrm{Na}_{2} \mathrm{SO}_{4}$ and concentrated. Crude material was purified by triturating with diethyl ether to get compound 3 .

\section{3-Chloro-N-(4-methoxyphenyl)-N-(prop-2-ynyl) quinoxalin-2-amine (3a)}

${ }^{1} \mathrm{HNMR}\left(400 \mathrm{MHz} \mathrm{CDCl}_{3}\right): \delta=7.92(\mathrm{~d}, 1 \mathrm{H}, \mathrm{J}=7.8 \mathrm{~Hz}, \mathrm{ArH}), 7.90(\mathrm{~d}, 1 \mathrm{H}, \mathrm{J}=7.8 \mathrm{~Hz}, \mathrm{ArH}), 7.67(\mathrm{t}, 1 \mathrm{H}, \mathrm{ArH}), 7.54(\mathrm{t}$, 1H, ArH), 7.09 (d, 2H, ArH), 7.09 (d, 2H, ArH), $6.90(\mathrm{~d}, 2 \mathrm{H}, \mathrm{ArH}), 4.69\left(\mathrm{~d}, 2 \mathrm{H}, \mathrm{J}=2.4 \mathrm{~Hz},-\mathrm{CH}_{2}\right), 3.82\left(\mathrm{~s}, 3 \mathrm{H},-\mathrm{CH}_{3}\right), 2.19$ $(\mathrm{t}, 1 \mathrm{H},-\mathrm{CH})$;

${ }^{13} \mathrm{C}$ NMR $\left(100 \mathrm{MHz}, \mathrm{CDCl}_{3}\right): \delta=158,149,141,139,138,138,130,127,127,127,114,79,72,72,55,55,43$; Mass: $\mathrm{m} / \mathrm{z}=325\left[\mathrm{M}^{+1}\right]$;

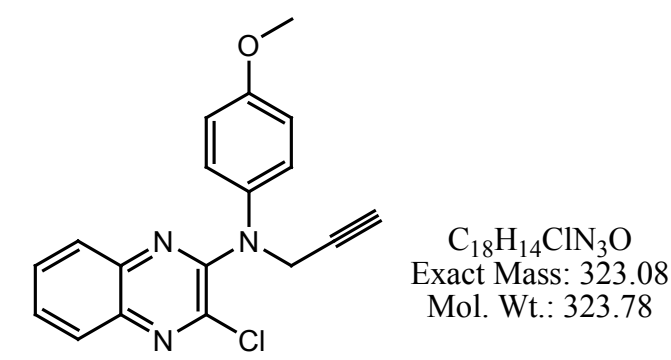

Figure 2. $\mathrm{C}_{18} \mathrm{H}_{14} \mathrm{ClN}_{3} \mathrm{O}$, exact mass: 323.08, Mol. Wt.: 323.78

\section{3-Chloro-N-(4-bromophenyl)-N-(prop-2-ynyl) quinoxalin-2-amine (3b)}

${ }^{1} \mathrm{HNMR}\left(400 \mathrm{MHz} \mathrm{CDCl}_{3}\right): \delta=7.94(\mathrm{~d}, 1 \mathrm{H}, \mathrm{ArH}), 7.91(\mathrm{~d}, 1 \mathrm{H}, \mathrm{ArH}), 7.72(\mathrm{t}, 1 \mathrm{H}, \mathrm{ArH}), 7.62(\mathrm{t}, 1 \mathrm{H}, \mathrm{ArH}), 7.49$ (d, 2H, ArH), $7.01(\mathrm{~d}, 2 \mathrm{H}, \mathrm{ArH}), 4.74\left(\mathrm{~d}, 2 \mathrm{H}, \mathrm{J}=2 \mathrm{~Hz},-\mathrm{CH}_{2}\right), 2.21(\mathrm{t}, 1 \mathrm{H},-\mathrm{CH})$;

${ }^{13} \mathrm{C}$ NMR $\left(100 \mathrm{MHz}, \mathrm{CDCl}_{3}\right): \delta=148,144,141,139,138,132,131,130,128,127,127,126,119,79,72,43$; Mass: $\mathrm{m}$ $/ \mathrm{z}=374\left[\mathrm{M}^{+1}\right]$;

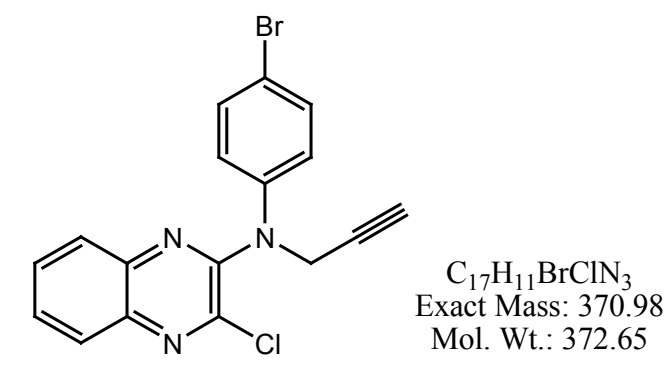

Figure 3. $\mathrm{C}_{17} \mathrm{H}_{11} \mathrm{BrClN}_{3}$, exact mass: 370.98, Mol. Wt.: 372.65

\section{3-Chloro-N-(4-chlorophenyl)-N-(prop-2-ynyl) quinoxalin-2-amine (3c)}

${ }^{1} \mathrm{HNMR}\left(400 \mathrm{MHz} \mathrm{CDCl}_{3}\right): \delta=7.93(\mathrm{~d}, 1 \mathrm{H}, \mathrm{ArH}), 7.90(\mathrm{~d}, 1 \mathrm{H}, \mathrm{ArH}), 7.72(\mathrm{t}, 1 \mathrm{H}, \mathrm{ArH}), 7.61(\mathrm{t}, 1 \mathrm{H}, \mathrm{ArH}), 7.48(\mathrm{~d}, 2 \mathrm{H}$, $\mathrm{ArH}), 7.00(\mathrm{~d}, 2 \mathrm{H}, \mathrm{ArH}), 4.73\left(\mathrm{~d}, 2 \mathrm{H}, \mathrm{J}=2.2 \mathrm{~Hz},-\mathrm{CH}_{2}\right), 2.20(\mathrm{t}, 1 \mathrm{H},-\mathrm{CH})$;

${ }^{13} \mathrm{C}$ NMR $\left(100 \mathrm{MHz}, \mathrm{CDCl}_{3}\right): \delta=148,144,141,139,138,132,131,130,128,127,127,126,119,79,72,43$; Mass: $\mathrm{m}$ $/ \mathrm{z}=329\left[\mathrm{M}^{+1}\right]$;

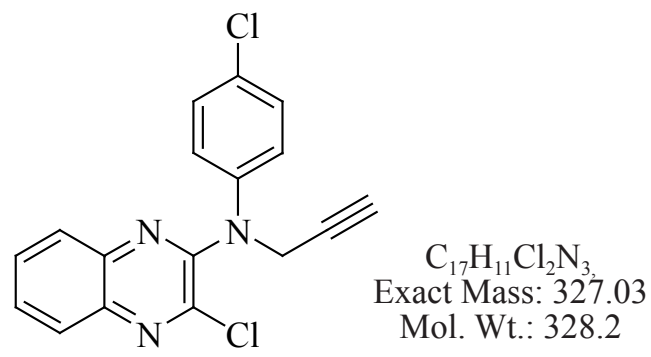

Figure 4. $\mathrm{C}_{17} \mathrm{H}_{11} \mathrm{Cl}_{2} \mathrm{~N}_{3}$, exact mass: 327.03 , Mol. Wt.: 328.2 
3-Chloro-N-(4-fluorophenyl)-N-(prop-2-ynyl) quinoxalin-2-amine (3d)

${ }^{1} \mathrm{HNMR}\left(400 \mathrm{MHz} \mathrm{CDCl}_{3}\right): \delta=7.93(\mathrm{~d}, 1 \mathrm{H}, \mathrm{ArH}), 7.90(\mathrm{~d}, 1 \mathrm{H}, \mathrm{ArH}), 7.70(\mathrm{t}, 1 \mathrm{H}, \mathrm{ArH}), 7.60(\mathrm{t}, 1 \mathrm{H}, \mathrm{ArH}), 7.50(\mathrm{~d}$, $2 \mathrm{H}, \mathrm{ArH}), 7.00(\mathrm{~d}, 2 \mathrm{H}, \mathrm{ArH}), 4.73\left(\mathrm{~d}, 2 \mathrm{H}, \mathrm{J}=2 \mathrm{~Hz},-\mathrm{CH}_{2}\right), 2.20(\mathrm{t}, 1 \mathrm{H},-\mathrm{CH})$;

${ }^{13} \mathrm{C}$ NMR $\left(100 \mathrm{MHz}, \mathrm{CDCl}_{3}\right): \delta=149,144,141,139,138,132,131,130,128,127,127,126,119,79,72,43$; Mass: $\mathrm{m}$ $/ \mathrm{z}=313\left[\mathrm{M}^{+1}\right]$

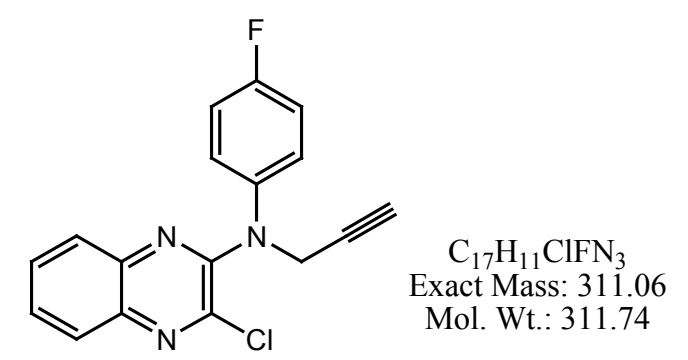

Figure 5. $\mathrm{C}_{17} \mathrm{H}_{11} \mathrm{CIFN}_{3}$, exact mass: 311.06, Mol. Wt.: 311.74

Step 3: General experimental procedure for the synthesis of ( 4 a-e $)$

To a solution of Compound 3, reaction mixture containing, p-tolyl sulfonyl azide $(0.36 \mathrm{mmol})$, terminal alkyne $(0.35 \mathrm{mmol})$ ortho-amino aniline $(0.33 \mathrm{mmol}), \mathrm{CuI}(0.03), \mathrm{MeCN}(1 \mathrm{~mL})$ and $\mathrm{Et}_{3} \mathrm{~N}(0.66 \mathrm{mmol})$ was added at room temperature and stirred for $1 \mathrm{~h}$. Then, added the con. $\mathrm{H}_{2} \mathrm{SO}_{4}(0.1 \mathrm{~mL})$. The resulting reaction mixture was subjected to microwave irradiation at $120^{\circ} \mathrm{C}$ for 20 min. Reaction mixture was diluted with cold water, basified with $\mathrm{K}_{2} \mathrm{CO}_{3}$ and extracted with EtOAc $(3 \times 5 \mathrm{~mL})$. The combined organic layer was washed with water, brine, dried over anh. $\mathrm{Na}_{2} \mathrm{SO}_{4}$ and concentrated. Crude material was purified by flash column chromatography over silica gel (100-200mesh) column chromatography using $50-70 \%$ EtOAc in pet-ether to get pure compound 4.

3-Chloro-N-(2(5-chloro-1-tosyl-1H-benzo [d] imidazol-2-yl)ethyl)-N-p-tolylquinoxalin-2-amine (4a)

${ }^{1} \mathrm{HNMR}\left(400 \mathrm{MHz} \mathrm{CDCl}_{3}\right): \delta=7.87(\mathrm{~d}, 1 \mathrm{H}, \mathrm{ArH}), 7.84(\mathrm{~d}, 1 \mathrm{H}, \mathrm{ArH}), 7.79(\mathrm{~d}, 1 \mathrm{H}, \mathrm{ArH}), 7.65(\mathrm{~m}, 3 \mathrm{H}, \mathrm{ArH}), 7.62(\mathrm{~d}$, $1 \mathrm{H}, \mathrm{ArH}), 7.55(\mathrm{~m}, 1 \mathrm{H}, \mathrm{ArH}), 7.14-7.01(\mathrm{~m}, 7 \mathrm{H}, \mathrm{ArH}), 4.56\left(\mathrm{t}, 2 \mathrm{H},-\mathrm{CH}_{2} \mathrm{~N}\right), 3.62\left(\mathrm{t}, 2 \mathrm{H},-\mathrm{CH}_{2}\right), 2.50\left(\mathrm{~s}, 3 \mathrm{H},-\mathrm{CH}_{3}\right), 2.34(\mathrm{~s}$, $\left.3 \mathrm{H},-\mathrm{CH}_{3}\right)$;

${ }^{13} \mathrm{C}$ NMR $\left(100 \mathrm{MHz}, \mathrm{CDCl}_{3}\right): \delta=161,159,151,149,145,142,141,139,139,139,135,135,133,129,127,127,126$, $126,126,126,125,119,116,113,52,27,21,21 ;$ Mass: $\mathrm{m} / \mathrm{z}=604\left[\mathrm{M}^{+1}\right]$;

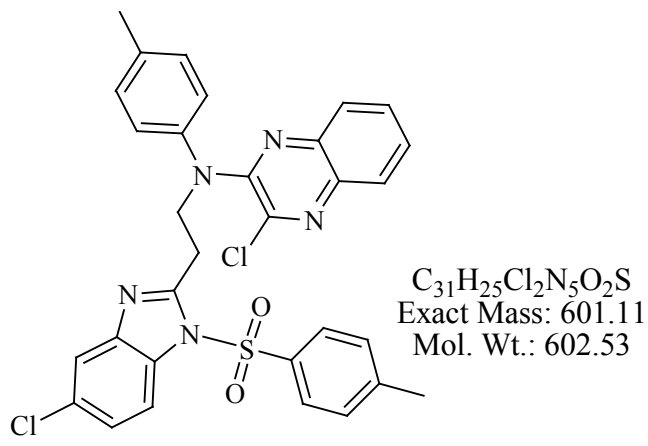

Figure 6. m/e: 601.11 (100.0\%), 603.11 (69.8\%), 602.11 (36.3\%), 604.11 (23.8\%), 605.10 (13.1\%), 603.12 (5.6\%), $605.11(4.6 \%), 606.11(4.6 \%), 604.10(1.3 \%)$ C, 61.79; H, 4.18; Cl, 11.77; N, 11.62; O, 5.31; S, 5.32

3-Chloro-N-(2-(5-chloro-1-tosyl-1H-benzo [d] imidazol-2-yl) ethyl)-N-(4methoxy phenyl) quinoxalin-2-amine (4b)

${ }^{1} \mathrm{HNMR}\left(400 \mathrm{MHz} \mathrm{CDCl}_{3}\right): \delta=7.91(\mathrm{~d}, 1 \mathrm{H}, \mathrm{ArH}), 7.88(\mathrm{~d}, 1 \mathrm{H}, \mathrm{ArH}), 7.78(\mathrm{~d}, 1 \mathrm{H}, \mathrm{ArH}), 7.69(\mathrm{~m}, 3 \mathrm{H}, \mathrm{ArH}), 7.60(\mathrm{~d}$, $1 \mathrm{H}, \mathrm{ArH}), 7.56(\mathrm{~m}, 1 \mathrm{H}, \mathrm{ArH}), 7.18-7.02(\mathrm{~m}, 7 \mathrm{H}, \mathrm{ArH}), 4.58\left(\mathrm{t}, 2 \mathrm{H},-\mathrm{CH}_{2} \mathrm{~N}\right), 3.77\left(\mathrm{~s}, 3 \mathrm{H},-\mathrm{CH}_{3}\right), 3.61\left(\mathrm{t}, 2 \mathrm{H},-\mathrm{CH}_{2}\right), 2.35(\mathrm{~s}$, $\left.3 \mathrm{H},-\mathrm{CH}_{3}\right)$;

${ }^{13} \mathrm{C}$ NMR $\left(100 \mathrm{MHz}, \mathrm{CDCl}_{3}\right): \delta=161,159,151,149,145,142,141,139,139,139,138,135,134,133,130,127,127$, $126,126,126,125,125,119,116,113,52,45,27,21 ;$ Mass: $\mathrm{m} / \mathrm{z}=620\left[\mathrm{M}^{+1}\right]$; 


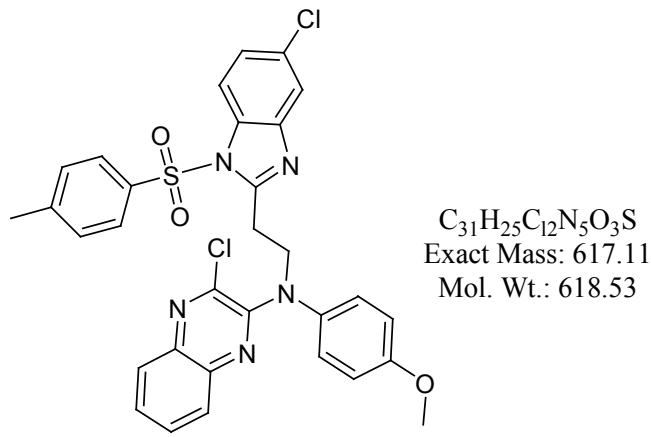

Figure 7. m / e: $617.11(100.0 \%), 619.10(68.5 \%), 618.11(33.9 \%), 620.11(22.1 \%), 621.10(13.6 \%), 619.11(7.0 \%)$, $622.10(4.8 \%), 621.11(4.4 \%), 620.10(3.3 \%), 618.10$ (2.6\%) C, 60.20; H, 4.07; Cl, 11.46; N, 11.32; O, 7.76; S, 5.18

3-Chloro-N-(2-(5-chloro-1-tosyl-1H-benzo [d] imidazol-2-yl) ethyl)-N-(4-bromophenyl) quinoxalin-2-amine (4c) ${ }^{1} \mathrm{HNMR}\left(400 \mathrm{MHz} \mathrm{CDCl}_{3}\right): \delta=7.95(\mathrm{~d}, 1 \mathrm{H}, \mathrm{ArH}), 7.91(\mathrm{~d}, 1 \mathrm{H}, \mathrm{ArH}), 7.79(\mathrm{~d}, 1 \mathrm{H}, \mathrm{ArH}), 7.70(\mathrm{~m}, 3 \mathrm{H}, \mathrm{ArH}), 7.65(\mathrm{~d}$, $1 \mathrm{H}, \mathrm{ArH}), 7.58(\mathrm{~m}, 1 \mathrm{H}, \mathrm{ArH}), 7.49$ (d, 2H, ArH), 7.20-7.01 (m, 5H, ArH), $4.56\left(\mathrm{t}, 2 \mathrm{H},-\mathrm{CH}_{2} \mathrm{~N}\right), 3.62\left(\mathrm{t}, 2 \mathrm{H},-\mathrm{CH}_{2}\right), 2.36(\mathrm{~s}$, $\left.3 \mathrm{H},-\mathrm{CH}_{3}\right)$;

${ }^{13} \mathrm{C}$ NMR $\left(100 \mathrm{MHz}, \mathrm{CDCl}_{3}\right): \delta=160,159,151,149,145,142,141,139,139,138,135,134,133,130,127,127,126$, $126,126,125,125,119,116,113,52,27,21$; Mass: $\mathrm{m} / \mathrm{z}=668\left[\mathrm{M}^{+1}\right]$;

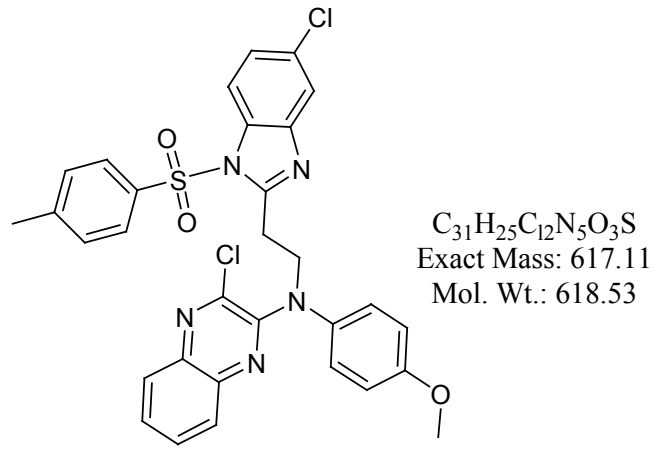

Figure 8. $\mathrm{m} / \mathrm{e}$ : $617.11(100.0 \%), 619.10(68.5 \%), 618.11(33.9 \%), 620.11(22.1 \%), 621.10(13.6 \%), 619.11(7.0 \%)$, $622.10(4.8 \%), 621.11(4.4 \%), 620.10(3.3 \%), 618.10(2.6 \%) \mathrm{C}, 60.20 ; \mathrm{H}, 4.07 ; \mathrm{Cl}, 11.46 ; \mathrm{N}, 11.32 ; \mathrm{O}, 7.76 ; \mathrm{S}, 5.18$

3-Chloro-N-(2-(5-chloro-1-tosyl-1H-benzo [d] imidazol-2-yl) ethyl)-N-(4-chlorophenyl) quinoxalin-2-amine (4d)

${ }^{1} \mathrm{HNMR}\left(400 \mathrm{MHz} \mathrm{CDCl}_{3}\right): \delta=7.96(\mathrm{~d}, 1 \mathrm{H}, \mathrm{ArH}), 7.91(\mathrm{~d}, 1 \mathrm{H}, \mathrm{ArH}), 7.80(\mathrm{~d}, 1 \mathrm{H}, \mathrm{ArH}), 7.69(\mathrm{~m}, 3 \mathrm{H}, \mathrm{ArH}), 7.66(\mathrm{~d}$, $1 \mathrm{H}, \mathrm{ArH}), 7.59$ (m, 1H, ArH), 7.49 (d, 2H, ArH), 7.19-7.02 (m, 5H, ArH), $4.51\left(\mathrm{t}, 2 \mathrm{H},-\mathrm{CH}_{2} \mathrm{~N}\right), 3.61\left(\mathrm{t}, 2 \mathrm{H},-\mathrm{CH}_{2}\right), 2.37(\mathrm{~s}$, $\left.3 \mathrm{H},-\mathrm{CH}_{3}\right)$;

${ }^{13} \mathrm{C}$ NMR $\left(100 \mathrm{MHz}, \mathrm{CDCl}_{3}\right): \delta=160,159,151,149,145,142,141,141,139,139,138,135,134,133,130,127,127$, $126,126,126,125,125,119,116,113,52,27,21$; Mass: $\mathrm{m} / \mathrm{z}=624\left[\mathrm{M}^{+1}\right]$;

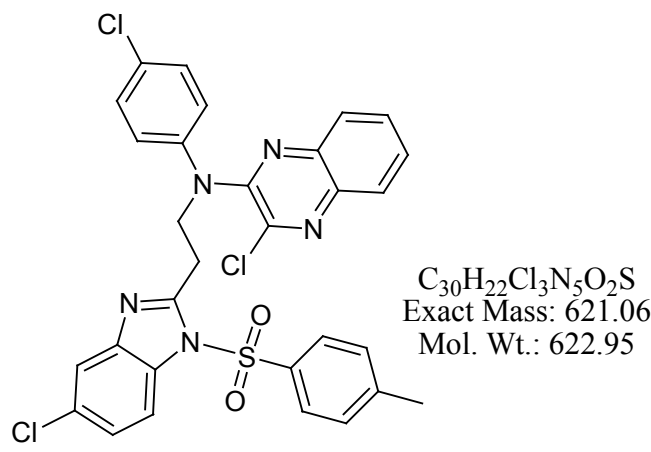

Figure 9. m/e: $623.05(100.0 \%), 621.06(99.6 \%), 625.05(35.5 \%), 622.06(33.4 \%), 624.06(33.1 \%)$ $626.05(12.2 \%), 623.06(6.4 \%), 625.06(5.9 \%), 627.05(5.1 \%), 624.05(2.6 \%), 627.06(1.9 \%)$, 622.05 (1.8\%), 628.05 (1.6\%) C, 57.84; H, 3.56; Cl, 17.07; N, 11.24; O, 5.14; S, 5.15 
3-Chloro-N-(2-(5-chloro-1-tosyl-1H-benzo[d]imidazol-2-yl) ethyl)-N-(4-fluorophenyl) quinoxalin-2-amine (4e) ${ }^{1} \mathrm{HNMR}\left(400 \mathrm{MHz} \mathrm{CDCl}_{3}\right): \delta=7.94(\mathrm{~d}, 1 \mathrm{H}, \mathrm{ArH}), 7.90(\mathrm{~d}, 1 \mathrm{H}, \mathrm{ArH}), 7.81(\mathrm{~d}, 1 \mathrm{H}, \mathrm{ArH}), 7.70(\mathrm{~m}, 3 \mathrm{H}, \mathrm{ArH}), 7.65(\mathrm{~d}$, $1 \mathrm{H}, \mathrm{ArH}), 7.60(\mathrm{~m}, 1 \mathrm{H}, \mathrm{ArH}), 7.50(\mathrm{~d}, 2 \mathrm{H}, \mathrm{ArH}), 7.21-7.03(\mathrm{~m}, 5 \mathrm{H}, \mathrm{ArH}), 4.52\left(\mathrm{t}, 2 \mathrm{H},-\mathrm{CH}_{2} \mathrm{~N}\right), 3.63\left(\mathrm{t}, 2 \mathrm{H},-\mathrm{CH}_{2}\right), 2.38(\mathrm{~s}$, $3 \mathrm{H},-\mathrm{CH}_{3}$;

${ }^{13} \mathrm{C}$ NMR $\left(100 \mathrm{MHz}, \mathrm{CDCl}_{3}\right): \delta=160,159,151,149,145,142,141,141,139,139,138,135,134,133,130,127,127$, $126,126,126,125,125,119,116,113,52,27,21 ;$ Mass: $\mathrm{m} / \mathrm{z}=607\left[\mathrm{M}^{+1}\right]$.

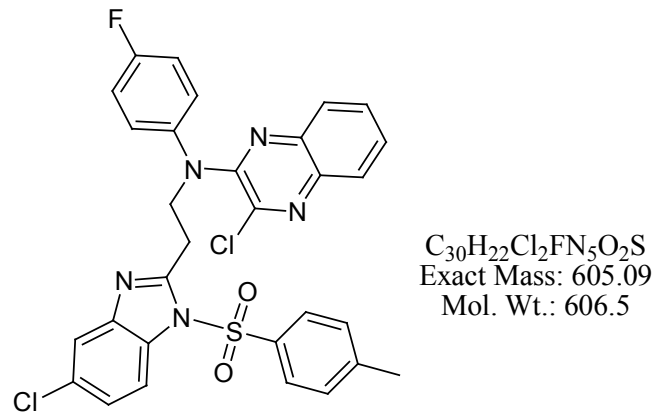

Figure 10. m/e: $605.09(100.0 \%), 607.08(68.5 \%), 606.09(32.8 \%), 608.09(21.3 \%), 609.08(13.6 \%), 607.09(6.4 \%)$, $610.08(4.6 \%), 609.09(4.0 \%), 608.08(3.2 \%), 606.08(2.6 \%)$ C, 59.41; H, 3.66; Cl, 11.69; F, 3.13; N, $11.55 ; 0,5.28 ;$ S, 5.29

\section{Conclusion}

Derivatives of 3-Chloro-N-(2-(5-chloro-1-tosyl-1H-benzo [d] Imidazol-2-yl) ethyl)-N-Substituted Quinoxalin-2Amine were successfully prepared from starting material DCQX. All the synthesized compounds were characterized by ${ }^{1} \mathrm{H}$ NMR, ${ }^{13} \mathrm{CNMR}$ and LCMS analytical methods. Authors have succeeded in using microwave irradiation.

\section{Acknowledgements}

Authors are thankful to management, Principal and Head, Department of Freshman Engineering, Geethanjali College of Engineering and Technology for their encouragement and support for doing the research work.

\section{References}

[1] Blaziak K, Danikiewicz W, Makosza M. How does nucleophilic aromatic substitution really proceed in nitro arenes? Computational prediction and experimental verification. J. Am. Chem. Soc. 2016; 138: 7276-7281.

[2] Galal SA, Abdelsamie AS, Tokuda $\mathrm{H}$ et al. synthesis, cancer chemo preventive activity and molecular docking study of novel quinoxaline derivatives. Eur. J. Med. Chem. 2011; 46: 327-340.

[3] Ammar YA, Al-Sehemi AG, El-Sharief AMS, El-Gaby MSA. Chemistry of 2,3-dichloroquinoxalines Phosphorus. Sulfur. Silicon. Relat. Elem. 2009; 184: 660-698.

[4] Jannyely Neri M, Renata $M$ et al. 2,3-Dichloroquinoxaline as a versatile building block for hetero aromatic nucleophilic substitution: A review of the last decade Ajani, O.O., Present status of quinoxaline motifs: Excellent path finders in therapeutic medicine. Eur. J. Med. Chem. 2014; 85: 688-715.

[5] Thompson, Verheij MHP, Van Muijlwijk Koezen JE, Lummis SCR, Leurs R, Esch IJP. Structure-activity relationships of quinoxaline-based 5-HT3 A and 5-HT3 AB receptor-selective ligands. Chem Med Chem. 2013; 8: 946-955.

[6] Miranda FS, Signori AM, Vicente J, de Souza B De, Priebe JP, Szpoganicz B, Gonçalves NS, Neves A. Synthesis of substituted di pyrido [3,2-a: $\left.2^{\prime} 3^{\prime}-\mathrm{c}\right]$ phenazines and a new heterocyclic di pyrido [3,2-f: 20,30-h] quinoxaline [2,3-b] quinoxaline. Tetrahedron. 2008; 64: 5410-5415.

[7] Prasad B, Kumar KS, Babu PV, Anusha K, Rambabu D, Kandale A, Vanaja GR, Kalle AM, Pal M. $\mathrm{AlCl}_{3}$ induced C-N bond formation followed by Pd / C-Cu mediated coupling-cyclization strategy: synthesis of pyrrolo [2,3-b] quinoxalines as anticancer agents. Tetrahedron Lett. 2012; 53: 6059-6066.

[8] Kumar KS, Rambabu D, Sandra S, Kapavarapu R, Krishna GR, Rao MVB, Chatti K, Reddy CM, Misra P, Pal M. $\mathrm{AlCl}_{3}$ induced (hetero) arylation of 2,3-dichloro quinoxaline: a one-pot synthesis of mono / di substituted quinoxalines as potential antitubercular agents. Bioorg. Med. Chem. 2012; 20: 1711-1722.

[9] Lauder K, Toscani A, Scalacci N, Castagnolo D. Synthesis and Reactivity of Propargyl amines in Organic Chemistry. Acs.Chem Rev. 2017; 24: 14091-14200.

[10] Naushad E, Yong Rok Lee. Cerium oxide nanoparticle-catalyzed three-component protocol for the synthesis of highly 
substituted novel quinoxalin-2-amine derivatives and 3,4-dihydroquinoxalin-2-amines in water. RSC Adv. $2014 ; 22$.

[11] Mota F, Gane P, Hampden-Smith K, Allerston CK, Garthwaite J, Selwood DL. A new small molecule inhibitor of soluble guanylate cyclase. Bioorg. Med. Chem. 2015; 23: 5303-5310.

[12] Luther, Rani CS, Suresh N, Rao MVB, Kapavarapu R, Suresh C, Babu PV, Pal M. Design and synthesis of novel indole-quinoxaline hybrids to target phospho diesterase 4 (PDE4). Arab. J. Chem. 2015; 1-10.

[13] Sunke R, Babu PV, Yellanki S, Medishetti R. Ligand-free MCR for linking quinoxaline framework with a benzimidazole nucleus: a new strategy for the identification of novel hybrid molecules as potential inducers of apoptosis. Organic \& Bio molecular chemistry. 2014; 6800-6805. 